\title{
Changes in dentofacial vertical dimension with class I before and after treatment orthodontic appliance with and without extractions of first four premolar teeth
}

\author{
Fitri Octavianti*, Jono Salim*, Bergman Thahar*, Endah Mardiati* \\ *Department of Orthodontics, Faculty of Dentistry Universitas Padjadjaran
}

\section{ABSTRACT}

Introduction: There are different opinions on the effect of extraction on the first four premolar teeth. The purpose of this study is to analyzed change of dentofacial vertical dimension before and after orthodontic treatment for two groups of patients treated with and without extractions of first four premolar teeth. Methods: A method by Sivakumar and Valiathan (2008) using lateral cephalometry on 20 extraction cases and 15 cases of non-extraction was employed. Tracing was conducted before and after orthodontic treatment and some 13 measurements were conducted manually using a protractor, i.e. mandibular plane angle, anterior facial height, lower anterior facial height, posterior facial height, facial height index, FH-U1, FH-U6, FL -L1, FL-L6, Svertical-U1, Svertical-U6, Pogvertical-L1-L6 Pogvertical. Statistical analysis using t-test with $\mathrm{P}>0.05$ for paired, Showed that there was no significant changes in dentofacial vertical dimension for malocclusion Class I cases before and after treatment for samples treated with and without extraction of the first four premolar teeth for both groups of samples. Results: No significant difference found in the dentofacial vertical dimension for the extraction and non-extraction groups of samples. Conclusion: orthodontic treatment for class I malocclusion cases, both with and without extraction, did not cause any significant difference in the dentofacial vertical dimension. Showed that there was no significant changes in dentofacial vertical dimension for class I malocclusion cases before and after treatment for samples treated with and without extraction of the first four premolar teeth for both groups of samples. Results: No significant difference found in the dentofacial vertical dimension for the extraction and non-extraction groups of samples. No significant difference found in the dentofacial vertical dimension for the extraction and non-extraction groups of samples. Conclusion: There are no changes in the vertical dimension of dentofacial before and after treatment in Class I with a retraction and extraction four first premolars fixed orthodontic appliance.

Keywords: Class I malocclusion, dentofacial vertical dimension, extraction, fixed orthodontics.

\section{INTRODUCTION}

Dental malocclusion is the state that deviates from normal occlusion. The cause is not a pathological process but because of deviations from malocclusion normal. Growth and development can be defined in various ways, one of them with the classification of Angle (1890s), which is based on the relationship between the Antero-posterior maxillary first molar and 
mandibular first molar, which first mesiobuccal maxillary molar cusp occlude the buccal groove of the mandibular first molar. Angle classification has malocclusion in three classes: class I, class II and class III. In Class I Angle the molar relationship is normal, but the line of occlusion is not right caused by malposition of teeth, rotation or other causes. Most of the patients have malocclusion with Class I Angle.2

Some researchers have reported the effects of orthodontic treatment on the vertical dimension. Orthodontic treatment by extraction can reduce the vertical dimension and increase the depth of the bite, especially when compared to treatment without extraction. 3 The results Cusimano and McLaughlin state that the extraction of the premolars does not change the vertical dimension, but in the cases of high angle, it can be fixed or slightly open.4 According Sivakumar and Valiathan (2008), staggers (1994), and Koradereli (1999) treatment with class I with or without revocation of four premolars not produce changes in the vertical dimension, but Chua (1993) found that revocation of the premolars first in class I not causing lower anterior facial height change significantly,

Hayasaki (2005) conducted a study with a sample of Japanese-Brazilians, found a similar change in the height of the face of the anterior and posterior facial height in the group without extraction of four first premolars in Class I and Class II division 1.9 The results from Kim on Koreans made up of 27 people with class I being treated by extraction of four second premolar, found no change in the vertical height dimension between before and after treatment except in the mandibular plane angle measurement and SN to palatal plane angle.10 The results Hui Cen (2005) in 15 patients with Class II division 1 with retrognathic mandible in the clinic PPDGS Orthodontic Padjadjaran University showed a positive correlation and is very strong and meaningful between the vertical dimension of the face of the lower anterior tooth and vertical dimensions of the maxillary incisor and bottom jaw and vertical dimensions maxillary molar. 11

Orthodontics at the University of Padjadjaran PPDGS clinic had never done research on skeletal vertical dimension, and the vertical dimension of the teeth with Class I. Based on the reasons stated above, the authors are interested in doing research on dento-facial vertical dimension in patients with Class I before and after treatment with Standard edgewise orthodontic appliance.

Treatment is done with and without the first premolar tooth extraction through lateral cephalometric photograph study approach using Sivakumar and Valiathan.he purpose of this study is to analyzed change of dentofacial vertical dimension before and after orthodontic treatment for two groups of patients treated with and without extractions of first four premolar teeth.

\section{METHODS}

This type of research is comparative analytic. The sample consisted of 35 lateral cephalometric photos before and after the treatment of adult patients who consist of men and women aged between 15 and 35 years who have a Class I treatment outcome with and without the extraction of four first premolars in the clinic PPDGS Orthodontics, the University of Padjadjaran between 2000-2010. Sample has a complete set of teeth until the first molars, Antero-posterior skeletal relationship $(\mathrm{ANB})=<00-<50$, having a vertical facial height $(\mathrm{SN}$ GoGn$)=<280-<360$ and overbite $=<0 \mathrm{~mm}-<6 \mathrm{~mm}$.

The method used is the vertical height measurement dentofacial method by Sivakumar

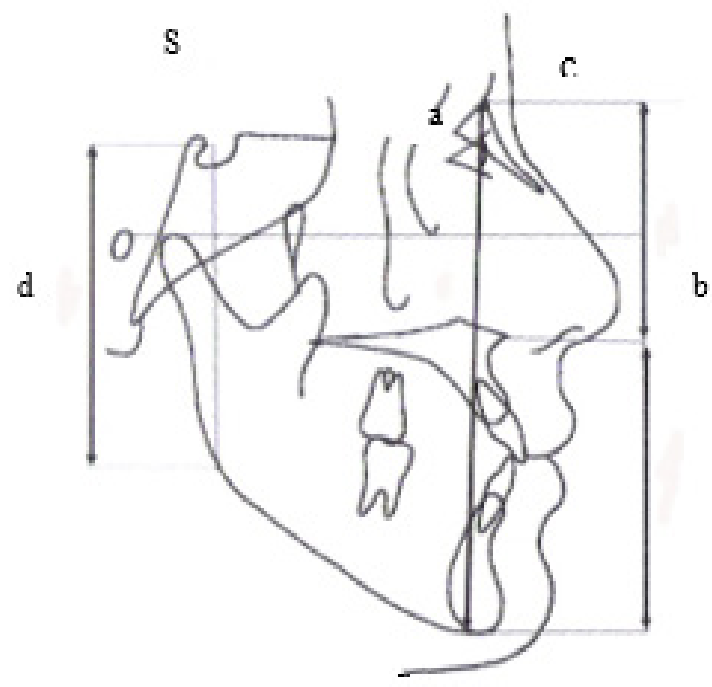

Figure 1. Vertical dimension methods of Sivakumar and Valiathan. ${ }^{5}$ A. Anterior face height (n-me, mm); B. Upper anterior facial height (n-ans $\mathrm{mm})$; C. Lower anterior facial height (ans-me, mm); D. Posterior face height (s-go, mm). ${ }^{10}$ 
and Valiathan consisting of skeletal vertical dimension and the vertical and horizontal dimensions of the teeth. The vertical skeletal dimension consists of plane angle of the mandible (SBM), height of the anterior face (TWA), height of the lower anterior face (TWAB), height of the posterior face (TWP), a high index face (ITW) and the vertical and horizontal dimension of the

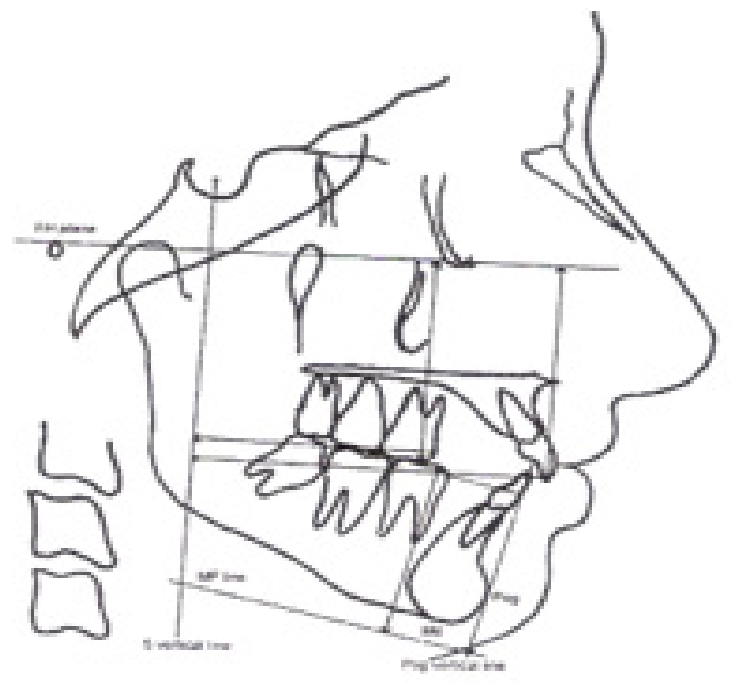

Figure 2.Vertical and horizontal dimension measurement methods of Sivakumar and Valiathan. ${ }^{5}$ Frankfort-i1: a. Maxillary incisor area. Frankfort-m1; b. Upper jaw area; c. Fiduciary line-i1 lower jaw; d. Fiduciary line-m1 lower jaw; e. Horizontal line distance i1 vertical- upper jaw; f. Horizontal distance of line-m1 vertical upper jaw; g. Vertical POG distance-i1 horizontal line of the lower jaw; h. Vertical POG line distance-m1 of the lower jaw. ${ }^{5}$ tooth consists of a Frankfort -I1 maxilla plane, plane-M1 Frankfort upper jaw, line-I1 lower jaw fiduciary, the fiduciary line-M1 lower jaw, distance of horizontal line I1 Svertikal- upper jaw, distance of horizontal line-M1 Svertikal upper jaw, the horizontal line spacing Pogvertikal-I1 lower jaw, distance of horizontal line Pogvertikal-M1 lower jaw. Analysis of data using the data test is paired with t-test $p<0.0 .5$.

\section{RESULTS}

The research data of dentofacial vertical analysis of 20 patients before and after the treatment in Class I were treated with the lifting of the four first premolars can be seen in Table 1.

From the 13 variables studied, there were noticeable changes in the skeletal dimensions, i.e. mandibular plane angle on average increased by $0.38^{\circ}$ (Std \pm 0.358 ), the average height anterior face is increased by $0: 28 \mathrm{~mm}$ (Std \pm 0.444 ), height of the anterior face below the average is increased by $0: 35 \mathrm{~mm}( \pm$ Std 0.366$)$, posterior facial height is increased by an average of 0:10 (Std \pm 0.455 ), high index faces increased by an average of 0:35 $\mathrm{mm}$ ( \pm Std 1.043). Changes to the dimensions of the teeth is the plane of Frankfort-I1 jaw on average increased by $0: 16 \mathrm{~mm}$ (Std \pm 0.690 ), plane of Frankfort-M1 jaw on average increased by $0.38 \mathrm{~mm}$ (Std \pm 0958 ), a line of fiduciary-11 lower jaw average increased by 0:06 mm ( \pm Std 0.531 ),

Table 1. The average results for dimensional measurement vertical dentofacial in class I treated with four teeth premolar revocation

\begin{tabular}{|c|c|c|c|c|c|c|c|}
\hline Variables & Before & Std & After & Std & Difference & Std & Nature \\
\hline Mandibular plane angle (0) & $23: 15$ & 1,514 & $23: 53$ & 1,585 & $0: 38$ & 0358 & Sig \\
\hline Anterior facial height (mm) & 115.70 & 2,953 & 115.98 & 2,808 & $0: 28$ & 0444 & Non Sig \\
\hline Lower anterior facial height & 64.60 & 2,030 & 64.95 & 1,966 & $0: 35$ & 0366 & Sig \\
\hline Posterior facial height (mm) & 75.01 & 3,244 & 75.11 & 3,149 & $0: 10$ & 0455 & Non Sig \\
\hline High index face & 69.99 & 3,395 & 70.34 & 3,411 & $0: 35$ & 1,043 & Non Sig \\
\hline FHP-I1 RA (mm) & 53.24 & 2430 & 53.40 & 2,198 & $0: 16$ & 0690 & Non Sig \\
\hline FHP-M1 RA (mm) & 46.88 & 3,153 & 47.25 & 3,301 & $0: 38$ & 0958 & Non Sig \\
\hline Fiduciary line-I1 RB (mm) & 44.76 & 1,633 & 44.83 & 1902 & 0:06 & 0531 & Non Sig \\
\hline Fiduciary-M1 line RB (mm) & 43.10 & 2,120 & 43.18 & 2232 & $0: 08$ & 0305 & Non Sig \\
\hline Svertical-I1 RA (mm) & 68.98 & 5499 & 66.39 & 5,133 & -2.59 & 1,483 & Sig \\
\hline Svertical-M1 RA (mm) & 39.99 & 3,738 & 40.73 & 3,806 & 0.74 & 0825 & Sig \\
\hline POGvertical-I1 RB (mm) & $4: 21$ & 1,996 & $2: 08$ & 1,489 & -2.14 & 1,108 & Sig \\
\hline POGvertical-M1 RB (mm) & -20.58 & 1,857 & -19.63 & 1,858 & 0.95 & 1,028 & Sig \\
\hline
\end{tabular}

Description: $\mathrm{ra}=\mathrm{rb}=$ maxilla mandible 
Table 2. The average results for dimensional measurement vertical dentofacial in class I treated without revocation four teeth premolar

\begin{tabular}{lccccccc}
\hline \multicolumn{1}{c}{ Variables } & Before & Std & After & Std & Difference & Std & Nature \\
\hline Mandibular plane angle $(0)$ & $22: 57$ & 1,280 & 22.80 & 1,251 & $0: 23$ & 0320 & Non Sig \\
Anterior facial height $(\mathrm{mm})$ & 119.67 & 5553 & 119.73 & 5,576 & $0: 07$ & 0221 & Non Sig \\
Lower anterior facial height & 66.30 & 3,206 & 66.38 & 3253 & $0: 08$ & 0181 & Non Sig \\
Posterior facial height $(\mathrm{mm})$ & 79.82 & 3,626 & 79.90 & 3588 & $0: 08$ & 0181 & Non Sig \\
High index face & 74.02 & 5,780 & 71.11 & 5,750 & $0: 09$ & 0481 & Non Sig \\
FHP-I1 RA $(\mathrm{mm})$ & 55.90 & 3,941 & 56.03 & 3,897 & $0: 13$ & 0508 & Non Sig \\
FHP-M1 RA $(\mathrm{mm})$ & 49.27 & 3,642 & 49.32 & 3566 & $0: 05$ & 0519 & Non Sig \\
Fiduciary line-I1 RB $(\mathrm{mm})$ & 45.67 & 2,717 & 45.48 & 2,899 & $0: 11$ & 0183 & Non Sig \\
Fiduciary-M1 line RB $(\mathrm{mm})$ & 44.20 & 3048 & 44.23 & 3,024 & $0: 03$ & 0326 & Non Sig \\
Svertical-I1 RA $(\mathrm{mm})$ & 71.70 & 3,890 & 72.77 & 3,766 & $1: 07$ & 1,419 & Sig \\
Svertical-M1 RA $(\mathrm{mm})$ & 41.55 & 2,597 & 41.65 & 2,582 & $0: 10$ & 0184 & Non Sig \\
POGvertical-I1 RB $(\mathrm{mm})$ & $1: 12$ & 1,145 & 1.67 & 1,148 & $0: 55$ & 0867 & Non Sig \\
POGvertical-M1 RB $(\mathrm{mm})$ & -21.83 & 1,984 & -21.75 & 1,973 & $0: 08$ & 0122 & Non Sig \\
\hline Description: ra $=\mathrm{rb}=\mathrm{maxilla}$ mandible & & & & & & &
\end{tabular}

Description: $\mathrm{ra}=\mathrm{rb}=$ maxilla mandible

Table 3. Analysis of differences test frequency distribution of the vertical dimension amendment dentofacial among group of revocation revocation by the group of four teeth without first premolar

\begin{tabular}{lccc}
\hline \multicolumn{1}{c}{ variables } & Std combined & thitung & Nature \\
\hline Mandibular plane angle $(0)$ & 0340 & $1: 32$ & Non sig \\
Anterior facial height $(\mathrm{mm})$ & 0350 & 1.88 & Non Sig \\
Lower anterior facial height & 0289 & 2.92 & not Sig \\
Posterior facial height $(\mathrm{mm})$ & 0346 & $0: 15$ & Non Sig \\
High index face & 0812 & $1: 04$ & Non Sig \\
FHP-I1 RA (mm) & 0605 & $0: 15$ & Non Sig \\
FHP-M1 RA (mm) & 0771 & $1: 33$ & Non Sig \\
Fiduciary line-I1 RB $(\mathrm{mm})$ & 0397 & -0.42 & Non Sig \\
Fiduciary-M1 line RB $(\mathrm{mm})$ & 0315 & $0: 42$ & Non Sig \\
Svertical-I1 RA (mm) & 1,451 & $3: 28$ & Sig \\
Svertical-M1 RA (mm) & 0598 & $3: 37$ & Sig \\
POGvertical-I1 RB $(\mathrm{mm})$ & 0995 & $5: 01$ & Sig \\
POGvertical-M1 RB $(\mathrm{mm})$ & 0732 & 3.74 & Sig \\
\hline
\end{tabular}

line-M1 lower jaw fiduciary average increased by 0:08 mm ( \pm Std 0.305), Research data analysis of vertical dento-facial of $\mathrm{I}$ to 15 patients before and after treatment in Class I were treated without the extraction of the four first premolars can be seen in Table 2.

From the 13 variables studied, there were noticeable changes in the skeletal vertical dimension, i.e. angle of the mandibular plane on average increased by $0.23^{\circ}$ (Std \pm 0.320 ), height of the anterior face in average increased by 0:07 $\mathrm{mm}$ (Std \pm 0.221 ), Height of anterior face belowaverage increased by 0:08 mm ( \pm Std 0.181), posterior facial height increased by an average of
0:08 (Std \pm 0.181 ), facial height index increased by an average of 0:09 $\mathrm{mm}( \pm$ Std 0.481$)$. Changes in the vertical dimension of the teeth is the plane of Frankfort-I1 jaw on average increased by $0: 13$ $\mathrm{mm}$ (Std \pm 0.508 ), plane of Frankfort-M1 jaw on average increased by $0.05 \mathrm{~mm}$ (Std \pm 0519 ), a line of fiduciary-I1 mandible the average increased by $0.11 \mathrm{~mm}( \pm$ Std 0.183), line-M1 lower jaw fiduciary average increased by 0:03 mm (Std \pm 0.326 ), Differences in the frequency distribution changes the dento-facial vertical dimension between the group without the .extraction of the first four premolars in Class I shows the results were not significant (Table 3) except in lower anterior 
facial height, the distance of the horizontal line 11 Svertikal- maxilla (Std combined 1.451, t calculate 3.28), the distance of the horizontal lines M1 Svertikal- maxilla (Std combined 0.598, t calculate 3:37), distance-I1 Pogvertikal horizontal line of the lower jaw (Std combined 0.995, t calculate 5:01), the distance of the horizontal line Pogvertikal-M1 lower jaw (std combined 0.732, t calculate 3.74).

\section{DISCUSSION}

Treatment with Class I can be done in two ways, namely by treatment using extraction method and treatment without extraction. Treatment with extraction is done to get room to fix the tooth in cases of severe crowding and to allow movement of the teeth in cases of incisor retraction to correct the protrusion. Treatment without extraction can be done with the expansion of the arch, slicing and disking..$^{1,12}$ In doing orthodontic treatment in Class I expect no changes in the vertical dimension. In this study, the vertical dimension of skeletal and dental is measured in 20 sample cases of extracted four first premolars and 15 sample cases without extraction of the four first premolars. The results from the analysis of paired data test showed that in Class $\mathrm{I}$, they were treated with extraction of four first premolar, before and after treatment there is no significant change except in the mandibular plane angle, lower anterior facial height, the distance of the horizontal line I1 Svertikal- upper jaw, line spacing Svertikal-M1 horizontal upper jaw, distance 11 Pogvertikal- horizontal line of the lower jaw, a horizontal line spacing Pogvertikal-M1 lower jaw.

The results of this research group that has undergone extraction discovered the additional average mandibular plane angle (SBM) is higher than the group without extraction as many as $0.38^{\circ}$ (Std \pm 0.358 ). The results of this study are smaller than the result of research done by Valiathan Sivakumar (2008), which is the addition of mandibular plane angles of $0.55^{\circ} .{ }^{5}$ According to English and Enlow ${ }^{12,13}$, the mandibular plane angle is an angle that is important for orthodontic treatment because it is associated with facial growth in a vertical pattern. The average height of the anterior face (TWA) in the treatment group before the extraction $115.70 \mathrm{~mm}$ ( \pm Std 2.953) and after treatment $115.98 \mathrm{~mm}$ ( \pm Std 2.808), the changes are not statistically significant. These results are consistent with Staggers research, Kocadereli and Sivakumar also Valiathan (2008) $.5,6,7$ Kocadereli study on the Turkish patients with Class I who are treated by extraction of four first premolars (40) Average of anterior facial height before treatment $123 \mathrm{~mm}( \pm$ Std 7:14) and after treatment $127.68 \mathrm{~mm}(\mathrm{Std} \pm 6.74) .7$ Increased anterior facial height can be caused by extrusion of molars or by closing the rest of the extracted space that cause extrusion of molars to increase the vertical dimensions. ${ }^{4}$

The results of orthodontic treatment with Class I with the extraction of the four first premolars reflect the addition of the lower anterior facial height (TWAB) averaging at 0.35 $\mathrm{mm}$ ( \pm Std 0366). Results of the study according to the study in infancy by Kocadereli which result in lower anterior facial height increase by an average of $4.67 \mathrm{~mm} \mathrm{(} \pm$ Std 5:05). ${ }^{7}$ Based on this research by Valiathan and Sivakumar which resulted in lower anterior facial height is average as much as $1: 33 \mathrm{~mm}( \pm$ Std 1:09).5 Vertical force that occurs during the closing of the space is essential for controlling the vertical dimension. unwanted vertical extrusive force on posterior teeth will increase lower anterior facial height. ${ }^{15}$

Measurement of average height in the posterior face in the extracted group before and after treatment changes $0: 10 \mathrm{~mm}$ (Std \pm 0.455 ) and high index faces change as much as 0:35 (Std $\pm 1,043$ ). Posterior face height is smaller than the result of Kocadereli with the addition of 3:53 $\mathrm{mm}$ ( \pm Std 4:36). ${ }^{7}$ The difference is likely due to differences in the sample used in Kocadereli research who are still in infancy.

Vertical dimension measurement in the set of teeth in the extracted group showed no significant change $(P<0.05)$. The results of plane measurements Frankfort-tooth incisor maxillary first as much as $0: 16 \mathrm{~mm}$ (Std \pm 0.690 ), plane Frankfort-first molar upper jaw as much as $0.38 \mathrm{~mm}$ (Std \pm 0958), a line of fiduciary-tooth incisor mandibular first as 0:06 mm (Std \pm 0.531 ), fiduciary-line mandibular first molar tooth as much as 0:08 mm ( \pm Std 0.305). Dimensional change of teeth-plane of Frankfort maxillary first molar tooth and the fiduciary-line mandibular first 
molar tooth can occur due to the extrusion of the upper molar teeth because their movements are not controlled. 4,9 $^{2}$ molar Frankfort-plane changes maxillary first incisor teeth and fiduciary-tooth line 1 mandibular incisor caused by mechanotherapy during treatment. ${ }^{4,10}$

The measurement results in the horizontal dimension of extracted group show a significant change in the distance of the horizontal line Svertikal-maxillary first incisor teeth, which before treatment $68.98 \mathrm{~mm}$ ( \pm Std 5.499) and after treatment of $66.39 \mathrm{~mm}( \pm$ Std 5.133), the distance of the horizontal line Svertikal- first molar upper jaw before treatment $39.99 \mathrm{~mm}$ (Std \pm 3738) and after treatment of $40.73 \mathrm{~mm}$ (Std $\pm 3,806$ ), the distance of the horizontal line Pogvertikal-tooth incisor mandibular first before treatment $4: 21 \mathrm{~mm}$ (Std \pm 1.996$)$ and after treatment 2:08 $\mathrm{mm}$ ( Std \pm 1,489 ), distance Pogvertikal- horizontal line of the mandibular molar teeth before treatment -20.58 $\mathrm{mm}( \pm$ Std 1857$)$ and after treatment $-19.63 \mathrm{~mm} \mathrm{(} \pm$ Std 1858). These changes can occur due to the loss of anchoring at the closing of the former extracted space to correct anterior tooth crowding, improve lip protrusion and the consequences are not exact. ${ }^{6,7,9}$

Analysis of data test paired vertical dimension dentofacial in Class I without the extraction of four first premolars showed no significant change $(p<0.05)$, except in the distance measurement horizontal line Svertikal-incisor 1 upper jaw before treatment $71.70 \mathrm{~mm}$ (Std \pm 3.890) and treatment after 72.77 ( \pm Std 3766). Changes in the vertical dimension skeletal, i.e. angle mandibular plane on average increased by 0.230 (Std \pm 0.320 ), height of the face anterior average increased by 0:07 $\mathrm{mm}$ (Std \pm 0221 ), Highface anterior below the average increased by $0: 08$ $\mathrm{mm}$ (Std \pm 0181$)$, posterior facial height increased by an average of 0:08 (Std \pm 0181 ), facial height index increased by an average of 0:09 $\mathrm{mm}( \pm$ Std 0481). The vertical dimension is the plane of Frankfort tooth-maxillary 11 average increased by 0:13 mm (Std \pm 0.508 ), plane of Frankfort-M1 jaw on average increased by $0.05 \mathrm{~mm}$ (Std \pm 0519 ), a line of fiduciary-l1 lower jaw on average increased by $0.11 \mathrm{~mm}$ (Std \pm 0183 ), a line of fiduciary-M1 lower jaw on average increased by 0:03 mm (Std \pm 0.326 ), the distance of the horizontal line of the maxillary I1 Svertikal- average increased by 1:07 $\mathrm{mm}( \pm$ Std 1419), the horizontal line spacing of the maxillary Svertikal-M1 increased by an average of 0:10 (Std \pm 0184), the distance of the line Pogvertikal horizontal jaw-I1 below the average increased by $0: 55 \mathrm{~mm}( \pm$ Std 0867), the distance of the horizontal line Pogvertikal-M1 lower jaw were reduced by an average of 0:08 $\mathrm{mm}( \pm$ Std 1122). These results are consistent with research Sivakumar and Valiathan, Cusinamo, staggers..$^{4,5}$

In this study, an analysis was also conducted to find out the difference in the frequency of the dento-facial vertical dimensions between the extraction group and the group without the removal of the first four premolar teeth. First premolars (table 4.3) except for the lower anterior face height, maxillary Svertical-I1 horizontal line distance, maxillary Svertical-M1 horizontal line distance, mandibular horizontal Pogvertical-I1 horizontal line distance, mandibular horizontal line distance Pogvertikal-M1. Changes occur more in the revocation group than in the non-revocation group. This result can be caused by the existence of undesired (uncontrolled) movements at the time of retraction of the anterior teeth to the posterior to close the former retraction space of the first four premolar teeth. Valiathan. ${ }^{5-7}$

\section{CONCLUSION}

There are no changes in the vertical dimension of dentofacial before and after treatment in Class I with a retraction and no retraction four first premolars with standard edgewise fixed orthodontic appliance.

\section{REFERENCES}

1. Graber TM, Vanarsdall RL. Orthodontics current principles and technique. 2nd ed. St Louis: Mosby Company; 1994. p. 64-75.

2. Proffit WR, Fields HW, Sarver DM. Contemporary orthodontics. 4th ed. Mosby Inc: St. Louis Philadelphia; 2007. p. 4, 201, 218-20, 282.

3. Schudy FF. The rotation of the mandible the resulting from growth: its implications in orthodontic treatment. Angle Orthod 1965; 35: 36-50.

4. Cusinamo C, McLaughlin RP. Effect of first bicuspid extraction on height in the high-angle cases. J Clin Orthod 1993; 27: 594-6. 
5. Sivakumar A, Valiathan A. Cephalometric assessment of vertical dentofacial changes in Class I subjects treated with and without extraction. Am J Orthod dentofacial Orthop 2008; 133; 869-75.

6. JA staggers. Vertical following chages first premolars extractions. Am J Orthod dentofacial Orthop 1994; 105: 19-24.

7. Koradereli I. The effect of first premolar extraction on vertical dimension. Am J Orthod dentofacial Orthop 1999; 116: 42-5.

8. AL Chua, Lim JY, Lubit EC. The effect of extraction versus nonextraction orthodontics treatment on the growth of the lower anterior face height. Am J Orthod dentofacial Orthop 1993; 104: 361-8.

9. Hayasaki BC. Influence of extraction and nonextraction orthodontic treatment in Japanese-Brazilians with class I and class
II division 1 malocclusions. Am J Orthod dentofacial Orthop 2005; 127: 30-6.

10. Kim TK. First or second premolar extraction effect on facial vertical dimension. Angle Orthodontist 2005; 75: 177-182.

11. Hui Cen. Relations lower anterior facial vertical dimension to the vertical dimension incisor and molar teeth under the Class II division I with mandibular retrognati absolute, [thesis]. Bandung: Unpad. 2005.

12. English JD, Peltomaki T, Pham K. Orthodontic review, Mosby: St.Louis; 2009. p. 53, 58-9.

13. Enlow DH. Handbook of facial growth. Philadelphia: WB Saunders Co; 1975. p. 264-5.

14. Wyatt NE. Preventing adverse effects on the temporomandibular joint through orthodontics treatment. Am J Orthod dentofacial Orthop 1987; 91: 493-9. 